\title{
Assessing mouse alternatives to access to computer: a case study of a user with cerebral palsy
}

\author{
Thais Pousada, Javier Pereira, Betania Groba, Laura Nieto, and Alejandro Pazos
}

Faculty of Health Science, Universidade da Coruna, A Coruna, Spain

\begin{abstract}
The purpose of this study is to describe the process of assessment of three assistive devices to meet the needs of a woman with cerebral palsy (CP) in order to provide her with computer access and use. The user has quadriplegic $\mathrm{CP}$, with anarthria, using a syllabic keyboard. Devices were evaluated through a three-step approach: (a) use of a questionnaire to preselect potential assistive technologies, (b) use of an eTAO tool to determine the effectiveness of each devised, and (c) a conducting semi-structured interview to obtain qualitative data. Touch screen, joystick, and trackball were the preselected devices. The best device that met the user's needs and priorities was joystick. The finding was corroborated by both the eTAO tool and the semi-structured interview. Computers are a basic form of social participation. It is important to consider the special needs and priorities of users and to try different devices when undertaking a device-selection process. Environmental and personal factors have to be considered, as well. This leads to a need to evaluate new tools in order to provide the appropriate support. The eTAO could be a suitable instrument for this purpose. Additional research is also needed to understand how to better match devices with different user populations and how to comprehensively evaluate emerging technologies relative to users with disabilities.
\end{abstract}

Keywords

Accessibility, Assistive technology, Computer access, Disability, Evaluation

\section{Introduction}

Information and communication technologies (ICTs) have redefined the way modern society operates (Harper, 2008). Computers are present in nearly every aspect of life, playing key roles in activities that include communications, information retrieval, and education, among many others (Blain, McKeever, \& Chau, 2010; Davies, Mudge, Ameratunga, and Stott, 2010). However, the level of computer use and the Internet by people with disabilities is still low when compared to that of the general population (Goodman, Jette, Houlihan, \& Williams, 2008). This may, in part, be due to the fact that individuals with disabilities need special methods and tools with which to access computers. These are collectively referred to as assistive technologies (ATs) or assistive devices. Particularly, assistive products in the area of ICTs are defined as "devices for helping a person receive, send, produce and/or process information in different forms" (Spanish Association for Standardisation and Certification, 2007, p. 10). Importantly, new avenues have been identified to assist individuals with special needs that can potentially provide them with the tools and resources needed to alleviate many of the traditional barriers encountered (Harper, 2008). 
One particular type of disability is that associated with cerebral palsy (CP). CP is defined as a group of permanent disorders affecting the development of movement and posture attributed to nonprogressive disturbances that occurred in the developing fetal or infant brain, which in turn place limitations on most types of activities (Rosenbaum, Paneth, Levinton, Goldstein, \& Bax, 2007). One of the main characteristics of CP is its variability.

It is important to take into account the fact that individuals with $\mathrm{CP}$ are usually not given the opportunity to use all AT devices that could allow them computer access. This may, in part, be due to the inherent complexity of the users' conditions (Hoppestad, 2007). For instance, one of the main problems is the difficulty experienced by such users in handling a mouse to perform simple operations with a computer: accurate pointing and clicking with a mouse can be a challenge for some users. Consequently, in such cases the selected AT must allow for several alternative pointing device combinations in order to address a large variety of physical impairments in persons with CP (Chen, Chu, Wu, \& Yeh, 2006).

In their review of 24 articles on the use of assistive devices and technologies by individuals with CP, Davies et al. (2010) concluded that a range of accessibility solutions are available for individuals with $\mathrm{CP}$, but most of these are in the early stages of development and there is little evidence of their effectiveness. However, an assistive device that works in one context may not be as effective in another (Cook \& Hussey, 2003; Cook \& Polgar, 2008). So, despite the wide range of available ATs, there seems to be an underutilization of them (Hoppestad, 2007). Among other factors (most of them social), the main problem could be derived by the poor assessment and delivery in selecting appropriate technologies and support resources.

In particular, selecting suitable strategies and devices is difficult for rehabilitation professionals primarily because of the lack of adequate assessment tools with which to evaluate a user's performance when interacting with the computer (Chen et al., 2006). Thus, AT practitioners need more effective methods to demonstrate the rationale behind their clinical decisions, as well as to assess the ultimate outcome of those decisions. (Koester, DiGiovine, Craik, \& Kneebone, 2007). Some assessment approaches are available for use in a rehabilitation context (Chen et al., 2006). However, none of the currently available methods has been widely adopted, and there seems to be a lack of a valid predictive model to guide device selection (Blain et al., 2010).

It is clear that evaluating a user's functional computer-operating performance is very important, as this practical information is vital in selecting the appropriate device to support the user's ability to interact with ICT in real situations (Chen et al., 2006). Furthermore, given the degree to which individualized modifications or innovations are required, some researchers argue that single-case studies may be the best approach to determine success for individuals with CP (Davies et al., 2010). This notion is in keeping with the broad concept of user-centred usability. With this in mind, this case report describes the process of assessment of three AT devices to meet the needs of a woman with athetoid $\mathrm{CP}$, in order to provide her with computer access and use. The three preselected devices are: touch screen, joystick, and trackball. To achieve this goal, a combination of three different evaluation methods was been employed: (a) a questionnaire-based approach, (b) use of a standardized tool (eTAO; Instituto Universitario de Integración en la Comunidad, 2008b), and (c) use of a semi-structured interview process. 


\section{Methods}

\section{Case Description}

The participant in this case (referred to as ARM) is a 41-year-old woman with quadriplegic choreoathetosis CP. She visits a CP center of A Coruña daily, the Asociación de Padres de Personas con Parálisis Cerebral de A Coruña (ASPACE), which currently provides services to 60 users.

ARM has no voluntary control of her four limbs, uses an electric wheelchair that she operates independently, and has involuntary movement in her arms and hands. According to the Manual Ability Classification System (MACS; Eliasson et al., 2006), she presents a level III, meaning that she can manipulate objects with difficulty if the activity has been previously organized and adapted. In addition, ARM presents anarthria, although she has relatively good oral and written comprehension levels. For instance, she is able to write large, complete phrases and uses a static syllabic communication board to communicate with others. She uses two fingers or the whole hand to select each syllable through a board, placed on a wheelchair support in front of her. Apart from this device, she also uses a dynamic syllabic communication board using the In-TIC program (author software) through an Apple iPad placed in the same support that static board (Pousada García et al., 2010).

ARM gave informed consent to participate in the work described here. In addition, the study was approved by the research ethics' code of the ASPACE center.

At present, ARM has a high level of motivation to use the computer, since it represents an important - and favorite - tool for carrying out various leisure and communications activities. Also, she uses the dynamic syllabic communicator as an alternative to a keyboard and to enhance the communication process.

Due to the limitations in general mobility, coordination of upper limbs, and motor dexterity, the subject has difficulty in adequately performing point-and-click operations with the mouse. As a result, it appears necessary to select an assistive device that would enable her access to, and effective use of, the mouse in order to provide her with a greater degree of independence and efficiency in that activity. This has led to an evaluation of her capacities and needs to select the best AT, based on her experience in its usage.

As part of the evaluation process, the user performed several mouse-based tasks under the supervision of rehabilitation professionals. It is important to point out that prior to conducting the assessment and selection of a specific device, ARM was unable to interact with a computer effectively using a standard mouse.

\section{Description of the Evaluation Methods and Tools}

\section{Questionnaire-Based Selection of Assistive Devices}

In order to determine the best assistive devices to facilitate the subject's access to and use of a computer, the computer access assessment (CAA) approach proposed by $\mathrm{Wu}, \mathrm{Men}, \mathrm{Wang}, \mathrm{Wu}, \&$ Li (2002) has been employed and expanded with the addition of several questions related to skills and abilities. This tool was selected as the best option because it quickly eliminates certain ATs that are unlikely to be usable by user.

The complete questionnaire was composed of four parts: (a) the subject's skills, (b) the subject's seating and positioning needs/preferences, (c) potential anatomical control site allocations, and (d) keyboard and mouse modifications necessary to meet the subject's needs. To evaluate each of these points, interviewers follows a simple algorithm that guides them until the 
following are determined: postural characteristics, potential anatomic control, and the selection of the most suitable support devices to assist the subject in using the computer.

\section{eTAO: Evaluation software tool}

The eTAO software was developed by the Instituto de Integración en la Comunidad (INICOUniversity of Salamanca) as tool with which to evaluate a user's functional capability to use a computer (inico.usal.es/etao; Instituto Universitario de Integración en la Comunidad, 2008b). The selection of this tool was based on a comparison of existing tools for evaluating assistive devices for computer usage. The comparison showed that only eTAO met the following criteria: it was developed and validated in Spain (thus adhering to legal, governmental, and cultural guidelines), it is written in the Spanish language, and is distributed online free of charge. So, eTAO is proposed as a tool more effective in our Spanish context.

This software was designed to facilitate assessing and monitoring the use of assistive devices that support information processing and communications tasks (Díez, Verdugo, Fernández, Campo, \& Velázquez, 2007). These tasks are based on two existing tools: assessment of computer task performance (ACTP; Mazer, Dumont, \& Vicent, 2003) and the software COMPASS (Koester, LoPresti, \& Simpson, 2006). In particular, eTAO consists of eight parts that help to evaluate a user's ability to perform mouse-related tasks (pointing and clicking) and keyboard-related tasks. The characteristics of each program, along with the associated evaluation factors, are shown in Table 1.

Table 1. Summary of eTAO tests

\begin{tabular}{|c|c|c|c|}
\hline Domain & Task & Description of task & Registered data of performance \\
\hline \multirow[t]{3}{*}{$\begin{array}{l}\text { Pointing } \\
\text { devices }\end{array}$} & $\begin{array}{l}\text { Objective-Click } \\
\text { and double click }\end{array}$ & $\begin{array}{l}\text { Move the cursor to an objective, } \\
\text { including clicking and double- } \\
\text { clicking on the target }\end{array}$ & $\begin{array}{l}\text { Result (success, error, and kind of error), reaction } \\
\text { time, inputs, wrong clicks, route }\end{array}$ \\
\hline & $\begin{array}{l}\text { Drag and } \\
\text { movement }\end{array}$ & $\begin{array}{l}\text { Drag an object from any location } \\
\text { to any destination }\end{array}$ & $\begin{array}{l}\text { Result (success, error, and kind of error), test time, } \\
\text { wrong clicks, drag attempts, lost opportunities, route }\end{array}$ \\
\hline & Menu & $\begin{array}{l}\text { Selection of any item on the menu } \\
\text { bar }\end{array}$ & $\begin{array}{l}\text { Result (success, error, and kind of error), total time, } \\
\text { time of menu and item, lost opportunities, successful } \\
\text { and wrong attempts. }\end{array}$ \\
\hline \multirow[t]{2}{*}{ Keyboard } & Letter & $\begin{array}{l}\text { Write any character (letter, number } \\
\text { or symbol) with keyboard }\end{array}$ & $\begin{array}{l}\text { Result (success, error, and kind of error), test time, } \\
\text { time of press, time of drop. }\end{array}$ \\
\hline & Word Sentence & $\begin{array}{l}\text { Write a word with keyboard Write } \\
\text { a sentence with keyboard }\end{array}$ & $\begin{array}{l}\text { Result (success, error, and kind of error), test time, } \\
\text { displacements, valid and non-valid segments, omitted } \\
\text { segments, speed of typing. }\end{array}$ \\
\hline \multirow[t]{2}{*}{$\begin{array}{l}\text { Single- } \\
\text { switch }\end{array}$} & Clicking & $\begin{array}{l}\text { Press and drop a switch. Maintain } \\
\text { the click during limited time }\end{array}$ & $\begin{array}{l}\text { Result (success, error, and kind of error), total time, } \\
\text { time of press, time of drop }\end{array}$ \\
\hline & Scanning & Selection through scanning system & $\begin{array}{l}\text { Result (success, error, and kind of error), total time, } \\
\text { planning time, scanning time, lost scanning. }\end{array}$ \\
\hline
\end{tabular}

The eTAO program is used online by accessing its website (Instituto Universitario de Integración en la Comunidad, 2008b). To use it, the professional is required to create a user account (free of charge). During the application of eTAO, the evaluator can administer all or a subset of the task, depending on the evaluation objectives. Previous results obtained using eTAO suggest that it (a) is statistically reliable, (b) is valid psychometrically as well as in terms of different environments, (c) offers a high degree of sensitivity, and (d) is easy to use (Díez et al., 2007). 
The subject was asked to participate in a semi-structured interview to obtain an optimal amount of information during the evaluation process. The semi-structured interview generated qualitative data, and was conducted after the eTAO-based evaluation of each device, in order to garner the subject's opinions about the tested devices.

\section{Assessment Process}

\section{Application of questionnaire to select the assistive devices}

The questionnaire (Appendix) was applied to determine the actual abilities as well as the postural, access, and usage needs of ARM. The obtained results permitted a preliminary selection of the subset of optimal assistive devices from those that were available, based on their potential for ease of use and effective control as preferred by the user. Based on these efforts, the selected devices were: (a) touch screen, (b) joystick, and (c) trackball.

So the preselection of these three ATs was motivated by the capacities and requirements of the participant. To follow with the next step on the assessment, these ATs were configured following the same criteria: buttons from joystick and trackball were set to the normal pointer speed configuration from Microsoft Windows system settings; clicking and double-clicking were established with eTAO tool, as will be discussed in the following section.

\section{Configuration of tasks for eTAO-based analysis}

In the second assessment, the main goal was to determine the appropriateness and adequacy of each preselected device and to facilitate the final selection of the optimal device for ARM. It should be noted that this analysis represents the major thrust of the investigations presented herein.

For that, the eTAO program was used (Instituto Universitario de Integración en la Comunidad, 2008b) and it was necessary to establish the format of each of the task to be performed prior to applying this tool. To this end, five mouse-related tasks were selected: (a) button-pressing, (b) clicking, (c) double-clicking, (d) moving, and (e) dragging (see Table 1 for descriptions of tasks). It should also be clarified that several tasks were discarded, including (a) those that evaluated the user's ability to use the keyboard (since ARM would use the dynamic syllabic keyboard), (b) those that evaluated menu usage (it is not deemed useful in the selection of an AT device), and (c) those that tested sweeping selection motions (since ARM does not use single-switch scanning).

Each task has certain configuration parameters that allow the evaluator to modify it according to both the user's abilities and limitations, and the final goals (Instituto Universitario de Integración en la Comunidad, 2008a).

The configuration of the characteristics of the five tasks was accomplished as follows:

Step 1: The goal of this phase was the configuration of the common parameters from eTAO, shown in Table 1 (Instituto Universitario de Integración en la Comunidad, 2008a). The same characteristics have been applied for all of the tasks (varying only the following values: name of the task, name of the configuration, and device).

Step 2: In this phase, the specific parameters were configured for each of the five preselected tasks. Table 2 shows these characteristics. 
Table 2. Configuration of specific characteristics

\begin{tabular}{|c|c|c|c|}
\hline Name of task & Description & Parameter & Configuration Characteristics \\
\hline \multirow[t]{7}{*}{ Button pressing } & $\begin{array}{l}\text { Assess the capacity to activate a switch in } \\
\text { response to any alert }\end{array}$ & Alert & Visual: Geometric figure \\
\hline & & Time of click & 0 seconds \\
\hline & & Time of pause & Constant \\
\hline & & Visual alert & Background color: Blue \\
\hline & & & Size: Big \\
\hline & & & Text: Click \\
\hline & & & Size of text: Medium \\
\hline \multirow[t]{6}{*}{$\begin{array}{l}\text { Clicking (move and } \\
\text { click on target) }\end{array}$} & $\begin{array}{l}\text { Assess the capacity to move the cursor to any } \\
\text { stimulate in different places of screen and make } \\
\text { click on the objective. }\end{array}$ & Type of objective & Geometric figure: Quadrate \\
\hline & & Method of selection & Click on objective \\
\hline & & Image & Clip art 1 \\
\hline & & Situation of objective & Percentages \\
\hline & & Distance & Large, medium, and short \\
\hline & & Draw route & Yes \\
\hline \multirow[t]{6}{*}{ Double clicking } & $\begin{array}{l}\text { Assess the capacity to make double click with the } \\
\text { cursor and to any stimulate in different places of } \\
\text { screen. }\end{array}$ & Type of objective & Geometric figure: Quadrate. \\
\hline & & Method of selection & Double click in objective \\
\hline & & Image & Clip art 1 \\
\hline & & Situation of objective & Percentages \\
\hline & & Distance & Large, medium, and short \\
\hline & & Draw route & Yes \\
\hline \multirow[t]{10}{*}{$\begin{array}{l}\text { Simple movement } \\
\text { (moving) }\end{array}$} & $\begin{array}{l}\text { Assess the capacity to move the cursor from any } \\
\text { localization to destination }\end{array}$ & Object & Type: Image \\
\hline & & & Size: Medium \\
\hline & & & Image: Clip art 1 \\
\hline & & Destination & Type: Image \\
\hline & & & Size: Medium \\
\hline & & & Image: Clip art 2 \\
\hline & & Method of selection & Click \\
\hline & & Localization & Percentages \\
\hline & & Background of screen & White \\
\hline & & Draw route & Yes \\
\hline \multirow[t]{10}{*}{ Dragging } & $\begin{array}{l}\text { Assess the capacity to drag an object from any } \\
\text { localization to destination and drop it. }\end{array}$ & Object & Type: Image \\
\hline & & & Size: Medium \\
\hline & & & Image: Clip art 1 \\
\hline & & Destination & Type: Image \\
\hline & & & Size: Medium \\
\hline & & & Image: Clip art 2 \\
\hline & & Method of selection & $\begin{array}{l}\text { Click with pointing in first } \\
\text { localization }\end{array}$ \\
\hline & & Localization & Percentages \\
\hline & & Background of screen & White \\
\hline & & Draw route & Yes \\
\hline
\end{tabular}




\section{Evaluation Based on the eTAO Program}

The eTAO-based evaluations of each of the three devices (touch screen, joystick, and trackball) were carried out over a consecutive three-day period, devoting one day to the evaluation of each device. The main reason for that was to give ARM enough time between assessments to rest and not to influence the results from each device. The evaluations were done in the computer classroom of the ASPACE Center using the same computer and by the same interviewer. ARM was placed in her wheelchair and in the correct position with respect to the computer screen and AT. During each session, a second researcher was present as an observer for the purposes of corroborating the evaluation process and its results.

To evaluate the effectiveness of each device, the five tasks (button-pressing, clicking, doubleclicking, moving, and dragging) were applied, allowing a 5-minute rest period between tasks. Prior to initiating each evaluation, the interviewer gave the user specific instructions regarding the development of the tasks and the actions to be performed.

\section{Semi-Structured Interview}

Once the five eTAO tasks were completed, the same interviewer posed four simple questions to the user. The responses to the questions were open-ended. The first four questions consisted of asking the user (a) to express her opinions or impressions regarding the tasks themselves (whether these tasks seemed long, arduous, reasonable, etc.), (b) the perceived degree of comfort while performing the tasks with each device, (c) the level of perceived fatigue, and (d) personal opinions or impressions regarding each device used. This fifth question consisted of three queries associated with (a) whether the device could be considered useful for using the computer, (b) the potential difficulties in using the device, and (c) possible modifications that might be implemented to improve the usability and efficacy of the device. As ARM presents anarthria, she used the static syllabic communicator to respond to the interview questions.

\section{Results}

\section{Requirements and Capacities of ARM: Preselecting AT}

The use of the questionnaire adapted by our research team made it possible to determine the abilities and limitations of the user. In addition, this method has uncovered insights regarding how to optimally select assistive devices for a specific user. The user demonstrated what might be viewed as residual functional mobility in the upper limbs when using the virtual keyboard, although the effective use of the mouse presented substantial difficulties for her. Thus, a preliminary selection of devices was made from all those currently available, reducing the options to (a) touch screen (pointing device with one-to-one correspondence between contact point and the underlying target application where the control is made by her finger), (b) joystick (the cursor moves in the corresponding relative direction, at a speed proportional to the device position), and (c) trackball (pointing device where the user makes relative movements to indicate corresponding).

\section{Results Using the eTAO Program}

Tables 3-7 summarize the evaluation results using the five aforementioned tasks with eTAO (button-pressing, clicking, double-clicking, moving, and dragging) for each of the three devices (touch screen, joystick, and trackball). 
Table 3. Results of assessment with eTAO_-button pressing.

\begin{tabular}{lcccc}
\hline Assessed assistive device & Success $(\%)$ & Errors $(1$ or 2) & No click $(\%)$ & Total required time (s) \\
\hline & & & & 0 \\
Touch screen & 100 & 0 & 0 & 2.28 \\
Joystick & 100 & 0 & 20 & 4.07 \\
Trackball & 70 & 10 (Error 2) & & \\
\hline
\end{tabular}

${ }^{a}$ Error 1: The person activated the button press, but he/she released it before completing the task. Error 2: The person clicked, but he/she did not release the button while performing the task.

\section{General Observations and Semi-Structured Interview}

\section{Touch screen}

The touch screen was one of the possible options offered to the subject for using the computer. This type of device is a highly intuitive for directly selecting icons shown on the screen. However, while performing tasks, it was observed that operating this device resulted in user tiredness and fatigue. ARM also had difficulties in completing the drag and movement tasks using this type of screen, which required compensatory motions: changing the arm (from the left to the right arm) to perform both tasks and seeking support for her upper limbs. Another factor contraindicating the use of the touch screen was the lack of precision, especially when performing the click and double-click tasks, which became clear in the results. The proportion of incorrect clicks was 30\%, while that of the incorrect double-clicks was 40\% (Tables 4 and 5). The observers noted that the positioning of the user at the wheelchair was not optimal during this evaluation, causing the user to adopt compensatory postures and experiencing poor back support. When conducting the semistructured interview, the user did not consider the tasks to be long or arduous. On the other hand, she thought that she felt quite tired during and after the eTAO-based evaluations, along with a low comfort level. With regard to personal perceptions, the user did not consider the touch screen to be an effective device for her, although she thought it might be for other users at the ASPACE Center.

Table 4. Results of assessment with eTAO_clicking.

\begin{tabular}{|c|c|c|c|c|c|c|}
\hline $\begin{array}{l}\text { Assessed assistive } \\
\text { device }\end{array}$ & $\begin{array}{l}\text { Success } \\
(\%)\end{array}$ & $\begin{array}{c}\text { Errors }(1 \text { or } 2)^{\mathrm{a}} \\
\quad(\%)\end{array}$ & $\begin{array}{l}\text { Selected objects with } \\
\text { wrong clicks }^{\mathrm{b}}(\%)\end{array}$ & $\begin{array}{l}\text { Time of } \\
\text { reaction }(s)\end{array}$ & $\begin{array}{c}\text { Time of } \\
\text { performance (s) }\end{array}$ & $\begin{array}{l}\text { Total time } \\
\text { (s) }\end{array}$ \\
\hline Touch screen & 50 & 20 (Error 2) & 30 & 2.25 & 3.51 & 5.76 \\
\hline Joystick & 100 & 0 & 0 & 2.29 & 5.13 & 7.42 \\
\hline Trackball & 80 & $\begin{array}{c}10 \text { (Error 1) } 10 \\
\quad(\text { Error } 2)\end{array}$ & 0 & 2.72 & 14.75 & 17.47 \\
\hline
\end{tabular}

${ }^{a}$ Error 1: The person clicked over objective, but he/she did not release it while performing the task. Error 2: The person did not select the objective because he/she did not put the cursor over it or not make click.

${ }^{\mathrm{b}}$ Wrong clicks: Number of unnecessary clicks or not valid clicks before successfully completing selection of the objective. 
Table 5. Results of assessment with eTAO—double click.

\begin{tabular}{|c|c|c|c|c|c|c|}
\hline $\begin{array}{l}\text { Assessed assistive } \\
\text { device }\end{array}$ & $\begin{array}{l}\text { Success } \\
(\%)\end{array}$ & $\begin{array}{l}\text { Errors }(1 \text { or } 2)^{\mathrm{a}} \\
\qquad(\%)\end{array}$ & $\begin{array}{l}\text { Selected objects with } \\
\text { wrong clicks }^{\text {b }}(\%)\end{array}$ & $\begin{array}{l}\text { Time of } \\
\text { reaction }(\mathrm{s})\end{array}$ & $\begin{array}{c}\text { Time of } \\
\text { performance (s) }\end{array}$ & $\begin{array}{l}\text { Total time } \\
\text { (s) }\end{array}$ \\
\hline Touch screen & 60 & 0 & 40 & 2.25 & 3.51 & 5.76 \\
\hline Joystick & 100 & 0 & 0 & 2.29 & 5.13 & 7.42 \\
\hline Trackball & 80 & $\begin{array}{l}10 \text { (Error 1) } 10 \\
\quad \text { (Error 2) }\end{array}$ & 0 & 2.72 & 14.75 & 17.47 \\
\hline
\end{tabular}

${ }^{a}$ Error 1: The person clicked over objective, but he/she did not release it while performing the task. Error 2: The person did not select the objective because he/she did not put the cursor over it or not make click.

${ }^{b}$ Wrong clicks: Number of unnecessary clicks or not valid clicks before successfully completing selection of the objective.

Table 5. Results of assessment with eTAO_-double click.

\begin{tabular}{|c|c|c|c|c|c|c|}
\hline $\begin{array}{l}\text { Assessed assistive } \\
\text { device }\end{array}$ & $\begin{array}{l}\text { Success } \\
(\%)\end{array}$ & $\begin{array}{c}\text { Errors }(1 \text { or } 2)^{\mathrm{a}} \\
(\%)\end{array}$ & $\begin{array}{l}\text { Selected objects with } \\
\text { wrong clicks }\end{array}$ & $\begin{array}{c}\text { Time of } \\
\text { reaction }(\mathrm{s})\end{array}$ & $\begin{array}{c}\text { Time of } \\
\text { performance }(\mathrm{s})\end{array}$ & $\begin{array}{l}\text { Total time } \\
\text { (s) }\end{array}$ \\
\hline Touch screen & 60 & 0 & 40 & 2.25 & 3.51 & 5.76 \\
\hline Joystick & 100 & 0 & 0 & 2.29 & 5.13 & 7.42 \\
\hline Trackball & 80 & $\begin{array}{l}10 \text { (Error 1) } 10 \\
\quad(\text { Error } 2)\end{array}$ & 0 & 2.72 & 14.75 & 17.47 \\
\hline
\end{tabular}

${ }^{a}$ Error 1: The person clicked over objective, but he/she did not release it while performing the task. Error 2: The person did not select the objective because he/she did not put the cursor over it or not make click.

${ }^{b}$ Wrong clicks: Number of unnecessary clicks or not valid clicks before successfully completing selection of the objective.

Table 6. Results of assessment with eTAO_moving.

Assessed assistive device Success (\%) Errors $^{\mathrm{a}}$ Time of test (s) Wrong clicks Attempts of drag Lost opportunities

\begin{tabular}{|c|c|c|c|c|c|c|}
\hline Touch screen & 70 & 30 & 8.5 & 0.1 & 1.95 & 0.45 \\
\hline Joystick & 90 & 10 & 6.3 & 0 & 1.15 & 0.15 \\
\hline Trackball & 70 & 30 & 8.2 & 0.3 & 1.50 & 0.40 \\
\hline
\end{tabular}

${ }^{a}$ Error 1: Number of test that the person did not complete the drag/movement during the established time.

${ }^{b}$ Number of times the person moved the cursor to the destination but he/she did not drop it to complete the drag. 
Table 7. Results of assessment with eTAO_-dragging.

\begin{tabular}{lcccccc} 
Assessed assistive device & Success $(\%)$ & Errors $^{\mathrm{a}}(\%)$ & Time of test (s) & Wrong clicks Attempts of drag & Lost opportunities $^{\mathrm{b}}$ \\
\hline & & & & & & \\
Touch screen & 40 & 60 & 9.8 & 0.6 & 2.30 & 1.90 \\
Joystick & 100 & 0 & 7.1 & 0 & 1.50 & 0.50 \\
Trackball & 60 & 40 & 9.1 & 0.4 & 1.85 & 1.80
\end{tabular}

${ }^{a}$ Error 1: Number of test that the person did not complete the drag/movement during the established time.

${ }^{b}$ Number of times that the person moved the cursor to the destination but he/she did not release it to complete the drag.

Joystick

During the evaluation on the use of the joystick, the screen was placed somewhat farther from the user relative to previous evaluation tests, and the joystick was placed on the workstation table, to one side of the keyboard.

As shown in the Tables 3-7, the joystick is the device that showed the best results in all of the tasks. The amount of time needed to complete the evaluation with this device was less.

With respect to the user's ability to control this device, the speed and skill of use were the best. It should be noted that the computer joystick works in a similar manner to the joystick control in the wheelchair. Moreover, the device has a built-in guard that helps locate the correct switch and avoids pressing the wrong keys. It also has a latching drag-switch and a double-click switch and provides a mechanism for controlling mouse speed movement. These characteristics allowed the user to move the mouse around and to handle its functions more effectively. In this sense, it is probably the control of the wheelchair by joystick that may have certain advantages to use this specific AT to access the computer.

The user's opinion regarding the use of the joystick was overall the most positive compared with the use of the other two devices evaluated. The tasks performed were the same as those performed with the touch screen; yet, as was the case with the touch screen, the user did not perceive the tasks as either long-lasting or arduous. Regarding comfort and fatigue, the perceived levels of both of these were comparatively high and low, respectively, in comparison with the touch screen. These findings were corroborated by the observations and comments made by the researchers.

It should also be noted that when the user was asked about the usefulness of the joystick, ARM explained that she considered it to be useful for controlling the computer. When asked about potential difficulties, as well as improvements, that she would suggest, she stated that the device ought to be situated at a lower height on the desk surface, such that she could be better able to handle the mouse without having to make compensatory movements.

\section{Trackball}

When the trackball was evaluated, the user had already become familiar with the tasks to be performed, during the previous two days. As the tables suggest (Tables 3-7), the time required to perform a task was three times as slow as those associated with the touch screen or the joystick(14.75 seconds execution time, 17.47 total time).

The control of trackball movements was viewed as generally good, although in two tasks (objective click-Table 4 and drag-Table 7) the user changed the effector hand (switching from left to right hand). In addition, it was observed that during the drag and drag-movement tasks, the user experienced some difficulty in moving the cursor toward the left side. 
Although ARM is left-handed, she used her right hand when activating the right button of the trackball, while using the left hand to press the left switch. A similar effect was observed during the drag action: when the user wanted to keep the left button pressed, she used her left hand, while her right hand was used to move over the trackball.

With respect to acquired posture during evaluation, it was observed that the user maintained an appropriate posture with a natural straight back. As a result, the movements appeared better proportioned.

The user's comments and opinions seem to support the results based on the eTAO program. The perceived level of fatigue while performing tasks was lower than that associated with the touch screen but higher than that associated with the joystick. In terms of perceived comfort, ARM stated that she felt comfortable in the workspace but not with the use of the trackball. Her overall impression of the device was that it is useful for her, but added, "I do not think that I can use it for a long time or in the long term."

\section{Global Results}

To check if the selected AT was the correct choice, a follow-up was applied. The research group observed the interaction between the participant and the computer with the new configuration two times per week for four weeks.

The selection of joystick allowed increasing not only the times per week in which she uses the computer (from five per week before assessment to nine per week), but also the efficacy and the efficiency of the participant during this activity. The obtained results from eTAO and from interview have established the adequate parameters to configure as the position and the buttons (speed and unlocked direction) of joystick.

Finally, the joystick was the device of election because it gave her the best access and usage of the computer, in comparison with the touch screen and trackball. The speed is faster in almost all tasks, the number of errors is low (30\% lower than trackball and 20\% lower than touch screen), and the percentage of success is $90 \%$ or higher in all task.

\section{Discussion}

This article describes an approach for selecting an assistive device to interact with a computer that is suitable and efficient for a user with disability. The focus is placed on reporting the results obtained in a specific case study in which a three-step approach with several device-selection methods was implemented to assess a female user afflicted with cerebral palsy.

\section{Computer Use as a Form of Social Participation and the Support From AT}

Computers are a basic form of social participation and interaction. Ideally, the computer (and the Internet) would thus serve as more than a simple tool but as a vital component in users lives that could support them in many activities that can directly and significantly broaden and improve their quality of life, particularly in terms of communicating and interacting with others (Shklovski, Kraut, \& Rainie, 2004).

In the case study presented here, it can be argued that if the selection of ATs had not been investigated for the user, she would have encountered difficulties in interacting with the computer through a standard mouse and it is likely that she would not have discovered many (and perhaps any) of these opportunities and resources. On the other hand, having the appropriate supportive tools and environment have clearly given the user completely new dimensions and abilities to 
improve her sense of personal autonomy, as well as opened up new avenues for decision-making and self-determination.

As suggested previously, ATs provide a means to circumvent barriers and increase the motivation to engage in diverse types of activities. There is compelling evidence to suggest that the abilities of a user otherwise limited by CP are enhanced or augmented by these technologies (Cook \& Hussey, 2003; Cook \& Polgar, 2008; Harper, 2008; Scherer et al, 2007). In agreement with Man and Wong (2007), a systematic and comprehensive approach should be carried out to assess the AT devices, including the end-user's needs, environmental factors, and levels of comfort or perceived effort.

In this case study, three AT devices were preselected based on their adequacy for a user with $\mathrm{CP}$ : touch screen, joystick, and trackball. Following both the eTAO-based tasks and the collection of ARM's opinions in the semi-structured interview, the joystick proved to be the tool that best adapted to her abilities, limitations, and needs. Compared to the conventional mouse, in ARM's case the joystick required very little effort to operate, while the trackball required significant effort (primarily pulling or sliding motions) to move the physical device. This suggests that it is very important to consider several types of devices when undertaking a device-selection process for a user with special needs. If the match is not an appropriate one from the viewpoint of the consumer, then the technology ought not to be used.

\section{The Importance of Evaluation Process to Select the Appropriate AT}

Prescribing the proper device to enable computer access to persons with disabilities is a complex process and no clear protocol has been developed to date. In general, the selection of a particular device should be based on what the individual user is able to do at the moment of evaluation, and not on what he or she might be able to do at a later stage (Cook \& Hussey, 2003; Cook \& Polgar, 2008). Nevertheless, the professional may also choose a device that will accommodate changes in the user's skills over time due to the progressive nature of some disabilities. It is clear that, in order to insure their effective use, assistive devices must be developed while addressing the real needs of end-users and while taking into account the cost of the device (Pousada, Pereira, Nieto, Groba, \& Pazos, 2011).

In addition, a good match between person and technology requires paying attention to the environment in which technology will be used, the needs and preferences of the user, and the functions and characteristics of the technology relative to these needs and preferences. (Scherer \& Craddock, 2002). Scherer et al. (2007) suggested the importance of developing a model to insure a successful selection process. The researchers consider the importance of ICF guidelines and, based on these guidelines, defined a framework for modeling the selection process. In this approach, two sets of factors are taken into account: personal factors and environmental factors (cultural and financial priorities; legislation and policy considerations; the perspectives of other persons significant to the user). Key features associated with this task are: a team-based approach, the role of the family, and evaluation components. The latter include actions such as establishing goals, task analysis, observations of how to function in daily environments, the activities of the individuals, and the characteristics and properties of assistive technology devices (Copley \& Ziviani, 2005). This would then be followed by a phase devoted to the development of a design of a plan for assistive intervention.

In this case study, a three-pronged approach has been taken to guide the evaluation process: (a) the use of a specific questionnaire focusing on the capabilities, the user's environment, and her goals, desires, needs, and priorities; (b) the use of standardized evaluation methods and techniques (e.g., eTAO) to evaluate the effectiveness of each AT devices considered; and (c) the development and implementation of a semi-structure interview designed to establish the opinions, perceptions, and preferences of the user with respect to each of the devices. In addition, the semi-structure interview indicates that the appropriate selection should ultimately lead to more satisfactory means with which to provide the user an improvement in his or her quality of life and overall well-being. 


\section{eTAO: A Tool to Assist in AT Device Selection}

As indicated by Barrelle et al. (1996), a key aspect of the evaluation process is the development and implementation of software tools with which to test performance in an objective manner, particularly in the case of users with disabilities. In a survey of the literature conducted by Díez et al. (2007), a wide variety of tools were identified for this purpose. These tools include: matching person and technology (MPT), the student, environment, task, and tools (SETT), and the Wisconsin assistive technology initiative (WATI). However, this review pointed out that most of these tools can be viewed as generic and lacking the specific focus on evaluating tools to assess AT devices that facilitate computer use (Díez et al., 2007). It seems that while some tools are available to assess a user's ability to use a computer, only two address the specific task of usercomputer interaction: assessment of computer task performance (ACTP; Dunn, 1991) and Compass software (Koester et al., 2006).

In contrast, eTAO seems to address most of these shortcomings, while also offering the desired degree of reliability and precision suitable for the purposes of the current case study (Díez et al., 2007). Furthermore, this program offers other advantages, including (a) the possibility to compare and evaluate different AT devices to enhance computer interaction; (b) the ability to identify potential difficulties with existing AT devices; (c) the option to register the evolution and changes in a user's abilities during the time he or she uses a particular device; (d) the ability to evaluate the effectiveness of assistive interventions using specific devices; (e) the capability to conduct a relative assessment of the user's skills in using AT devices relative to control groups; and (f) the support for undertaking investigative studies to assist in determining the degree to which a successful match might occur between user and device. These advantages from the application of eTAO have directly enhanced the assessment process in this particular case study.

However, during the eTAO-based evaluation process, two shortcomings were found that deserve consideration. One is the lack of a simpler language for both configuring tasks and showing the associated test results. Another is that the practicioner is currently unable to stop the tasks if necessary (e.g., when the user is disoriented or in the presence of distractions). It is hoped that future versions of the program can successfully address these shortcomings.

In spite of these limitations, and given its positive features, this program is considered to be a valid and useful tool for evaluating the user's abilities and for the selection of an appropriate AT device. We, therefore, encourage the translation of this tool to other languages and that it be implemented in other environments to further assess its reliability and usefulness.

\section{User-Centered Device Selection}

As previously mentioned, another key aspect in the selection process is the user's opinions and preferences based on results from semi-structured interview. This is perhaps best integrated in the selection process by making the user a primary "participant" in the process, since it enhances not only the selection itself but also the continued use of the selected ATs (Cook \& Hussey, 2003; Cook \& Polgar, 2008; Scherer \& Craddock, 2002). According to Louise-Bender, Kim, and Weiner (2002), successful integration of ATs into users' daily lives requires potential users to explore using the devices. This exploration reveals a number of things, including (a) how users "relate" to the devices, (b) the meanings they assign to them, (c) their expectations associated with using the technology, and (d) anticipated social costs. This points to the importance of understanding not only the user's disabilities but — what may be equally important — the user's own identity in relation to the technology (Louise-Bender, Kim, \& Weiner, 2002). In sum, personal factors influence the ability and willingness to perform or participate in certain activities as much as, if not more than, environmental factors. Personal factors determine the way in which users may value their physical, cognitive, and interpersonal capabilities and skills; these factors are likewise strongly related to the ways in which different technologies can maximize these capabilities. The interconnections between these factors and a particular technology play a central role in determining whether the technology is successfully incorporated in users' daily lives (Louise-Bender et al., 2002). 
With respect to the use of AT to enhance ICT, the importance of personal factors is also determinant. As shown in a survey done by Pousada et al. (2010), the responses of patients regarding the level of satisfaction with the use of computers were positive in all cases (very good or good), demonstrating the growing interest in new technologies and their possibilities as educational resources (Park \& Lee, 1996). More specifically, qualitative data can help practitioners to understand the effect of different factors, such as the expectations, preferences, needs, and priorities of the user; abilities and limitations; and resources (Chen et al., 2006; Scherer et al., 2007).

As it was seen in this case study, the user's opinions and preferences indeed played a determinant role in the AT selection process. The obtained information from semi-structured interview complemented and corroborated the results obtained with the eTAO-based studies.

Given the importance of each user's specific needs, priorities, and preferences in the selection process, it thus seems wise to incorporate a qualitative component in that. This can be accomplished through personal interviews such as the semi-structured interview used in this case study.

\section{Limitations of the Study}

This research was carried out as a case study of one specific subject and the assistive intervention to help the subject in interacting with a computer. Therefore, findings must be interpreted with caution.

The participant, ARM, is afflicted with athetotic CP. As such, she can provide insights into the types of technologies that can be useful to individuals that share a similar condition. However, it should be noted that there is significant heterogeneity in persons afflicted with CP in terms of physical, cognitive, and sensorial abilities. For this reason, it is essential to understand the specific needs of each user with this pathology, and to undertake comparative studies between different groups with different diagnostic profiles. It may be necessary to undertake assistive intervention efforts that are tailored specifically to each case. In this regard, additional research is needed to develop more comprehensive methods that can assess the possible psychosocial impact of computer usage (through the use ATs) on users with similar disabilities.

Another point to take account is the fact that the three devices were each tested once on separate days. So, the order or the experience with test could have an effect on the subjective data. At the same time, there are different models of trackballs and joysticks on the market. The assessment was done with available AT in ASPACE Center, so the procedure showed here would be applied with other types of trackballs and joysticks to determine if obtained results are the same, in comparison with these other devices.

Finally, it is observed that the evaluation process has been carried out in a specific environment: the computer classroom in the ASPACE Center. A particular environment can be associated with such influential factors as the available resources, support mechanisms, and interpersonal relationships; specific sets of behaviors, policies, and services; and other contextual parameters that can determine AT selection and usage.

Thos case study is a first step toward considering a user-centered approach to assess the selection of AT to access to computer. The results showed here are a sample that how a complete process consider not only the specific characteristics of devices, but also the needs and capacities of user and trying the effectiveness of these AT through a eTAO tool that will be able to apply for future research to improve its psychometric properties. 


\section{Conclusions}

This case report has been centered on examining the selection process that is required to select the optimal AT device to help the user with CP. It was based on a comprehensive method that integrates different tools and techniques. After the whole assessment process, the best device to meet user's needs and priorities was the joystick. The finding was corroborated by both eTAO tool and semi-structured interview.

This study has demonstrated that a reliable, precise, and efficient AT evaluation process consists of three phases: (a) a preliminary selection of candidate devices using the data derived from a specific questionnaire, (b) implementation of a standardized evaluation software tool (eTAO), and (c) the qualitative information and insights obtained through a semi-structured interview.

The study corroborates the hypothesis that AT device selection and user-device matching are significantly enhanced by incorporating a combination of factors, including (a) an assessment of the capabilities, limitations, and usage goals on the part of the user; (b) inclusion and participation of the user in the evaluation process (through interview); (c) a methodical evaluation of the functionality, advantages, disadvantages, and potential of preselected AT devices relative to the individual user; and (c) the environmental factors that can influence the user-device matching process and the eventual usage of the selected device. The method showed here is not the only one available assessment methodology, but the research group has demonstrated that the combination of different tools with the user's perspective is effective to determine the AT with the most adequate properties to meet ARM's needs.

The appropriate access to, and interaction with, a computer can significantly facilitate the user's ability to integrate socially while also being able to explore new avenues for participating in activities that may not otherwise be available to this base of users. However, although numerous assistive devices are currently available those allow users with CP to use computers, very few tools are available to actually assess these technologies. Therefore, future research efforts should consider the development and assessment of such instruments to determine their specific characteristics.

\section{References}

Barrelle, K., Laverty, W., Henderson, R., Gough, J., Wagner, M. and Hiron, M. 1996. User verification through pointing characteristics: An exploration examination. International Journal of Human-Computer Studies, , 45: 47-57.

Blain, S., McKeever, P. and Chau, T. 2010. Bedside computer access for an individual with severe and multiple disabilities: A case study. Disability and Rehabilitation: Assistive Technology, 5: 359-369.

Chen, M.-C., Chu, C.-N., Wu, T.-F. and Yeh, C.-C. 2006. "Computerized assessment aproach for evaluating computer interaction performance". In Proceedings of 10th Intranational Conference ICCHP: Computers Helping People with Special Needs (pp.450-456). Berlin: Springer-Verlag.

Cook, A. M. and Polgar, J. M. 2008. Assistive technologies: Principles and practice, 3rd, St. Louis, MO: Mosby.

Copley, J. and Ziviani, J. 2005. Assistive technology assessment and planning for children with multiple disabilities in educational settings. British Journal of Occupational Therapy, 68(12): 559-566.

Davies, T. C., Mudge, S., Ameratunga, S. and Stott, N. S. 2010. Enabling self-directed computer use for individuals with cerebral palsy: A systematic review of assistive devices and technologies. Developmental Medicine and Child Neurology, 52: 510-516.

Díez, E., Verdugo, M. A., Fernández, A., Campo, M.Velázquez, D.2007Diseño y validación de un sistema informatizado de evaluación de la capacidad funcional de personas con discapacidad en tareas de acceso al ordenador. Retrieved from http://www.imserso.es/InterPresent1/groups/imserso/documents/binario/idi132_06usalamanca.pdf

Dunn, W. 1991. “Assessing sensory performance enablers”. In Occupational therapy: Overcoming human performance deficits, 1 st ed., Edited by: Christiansen, C. and Baum, C. New York, NY: SLACK. InEds. 
Eliasson, A. C., Krumlinde-Sundholm, L., Rosblad, B., Beckung, E., Arner, M. and Ohrvall, A. M., \& Rosenbaum, P. 2006. The manual ability classification system (MACS) for children with cerebral palsy: Scale development and evidence of validity and reliability. Developmental Medicine and Child Neurology, 48: 549-554.

Goodman, N., Jette, A. M., Houlihan, B. and Williams, S. 2008. Computer and internet use by persons after traumatic spinal cord injury. Archives of Physical Medicine and Rehabilitation, 89: 1492-1498.

Harper, S. 2008. Computers and accessibility. Disability and Rehabilitation: Assistive Technology, 3(1-2): 1

Hoppestad, B. S. 2007. Inadequacies in computer access using assistive technology devices in profoundly disabled individuals: An overview of the current literature. Disability and Rehabilitation: Assistive Technology, 2: 189-199.

Instituto Universitario de Integración en la Comunidad. 2008a. eTAO: Evaluación para el uso de tecnologías de ayuda de acceso al ordenador. Manual de uso, 1st, Spain: Universidad de Salamanca. ed.). Salamanca

Instituto Universitario de Integración en la Comunidad2008bEvaluación para el uso de tecnologías de ayuda de acceso al ordenador Retrieved from http://inico.usal.es/etao (http://inico.usal.es/etao)

Koester, H., DiGiovine, C., Craik, S. A.Kneebone, M.2007Gathering and applying evidence in computer access services Retrieved from http://www.arata.org.au/download/koester_compass_article_20080117.doc

Koester, H., LoPresti, E. and Simpson, R. 2006, June. "Measurement validity for compass assessment software". In Presented at the 29th Annual Conference on Rehabilitation Engineering (RESNA) Atlanta, GA

Louise-Bender, P. T., Kim, J. and Weiner, B. 2002. The shaping of individual meanings assigned to assistive technology: A review of personal factors. Disability and Rehabilitation, 24: 5-20.

Man, D. W. and Wong, M. S. 2007. Evaluation of computer-access solutions for students with quadriplegic athetoid cerebral palsy. The American Journal of Occupational Therapy: Official Publication of the American Occupational Therapy Association, 61: 355-364.

Mazer, B., Dumont, C. and Vicent, C. 2003. Validation of the assessment of computer task performance. Technology and Disability, 15: 35-43.

Park, K. S. and Lee, K. T. 1996. Eye controlled human/computer interface using the line-of-sight and the intentional blink. Computers \& Industrial Engineering, 30: 463-473.

Pousada García, T., Nieto Rivero, L., Pereira Loureiro, J, Groba González, B., Moreiras Lorenzo, A., Mourelos Sánchez, I. and Pazos Sierra, A. 2010, March. "The I-TIC project. new software to support AAC". In Presented at the 25th Annual International Technology \& Persons with Disabilities Conference (CSUN) San Diego, CA.

Pousada, T., Pereira, J., Nieto, L., Groba, B. and Pazos, A. 2011. The use of computers and augmentative and alternative communication devices by children and young with cerebral palsy. Assistive Technology, 23(3), 135-149.

Rosenbaum, P., Paneth, N., Levinton, A., Goldstein, M. and Bax, M. 2007. The definition and classification of cerebral palsy. Developmental Medicine and Child Neurology, 49(S109): S1-S44.

Scherer, M. J. 2007. A framework for modeling the selection of assistive technology devices (ATDs). Disability and Rehabilitation: Assistive Technology, 2: 1-8. Jutai, J., Fuhrer, M., Demers, L. \& DeRuyter, F.

Scherer, M. J. and Craddock, G. 2002. Matching person \& technology (MPT) assessment process. Technology and Rehabilitation, 14: 125-131.

Shklovski, I., Kraut, R.Rainie, L.2004The internet and social participation: Contrasting cross-sectional and longitudinal analyses. Retrieved from http://jcmc.indiana.edu/vol10/issue1/shklovski_kraut.html

Assistive technology for people with disability. Classification and terminology, Spanish Association for Standardisation and Certification. (2007). UNE-EN ISO 9999:2007.

Wu, T.-F., Meng, L.-F., Wang, H.-P., Wu, W.-T. and Li, T.-Y. 2002, July. Computer access assessment for persons with physical disabilities: A guide to assistive technology interventions. Presented at the 8th International Conference ICCHP: Computers Helping People with Special Needs, Linz, Austria, 


\section{Appendix: Needs and Skills Questionnaire}

Assessing the Person's Skills

Skills Assessment

Sensory: visual and auditory function

Does the person have the skill?

Yes No With difficulty

Objects' recognition

Real pictures' recognition

Pictograms and symbols' recognition

Visual tracking

Auditory attention

Auditory discrimination

Perception of conversation with average volume

Physical skills

Fine motor skill

Gross motor skill

- Shoulder flex-ext

- Shoulder abd-add

- Elbow Flex-Ext

- Forearm pronosupination

- Wrist: rotation

- Neck: Flex-ext

- Neck: Lateral ext.

- Neck: rotation

Coordination eye-hand

Bilateral coordination

Cognitive skills

Memory

Categorization

Sequencing

Solve problems

Attention level

Communication Skills

Comprehension of oral message

Understanding of simple commands

Oral expression

Enough degree of interaction

Reading ability

Recognition of letters

Writing ability 
Assessing the Needs for Seating and Positioning

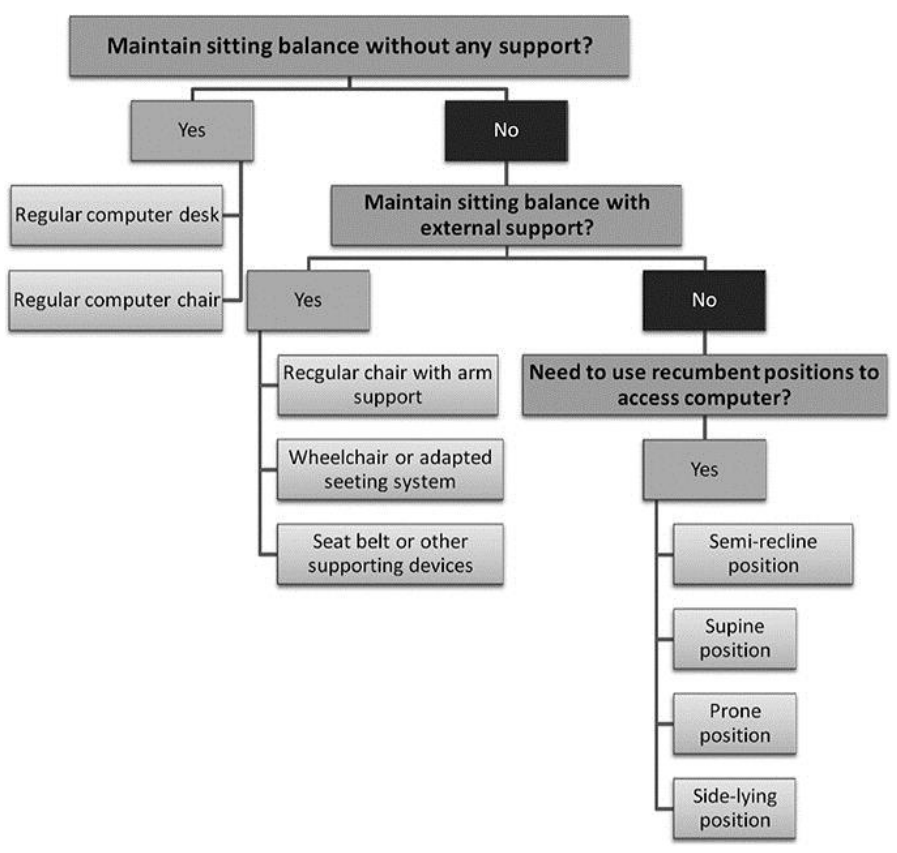

Determining Potential Anatomical Control Site Allocations

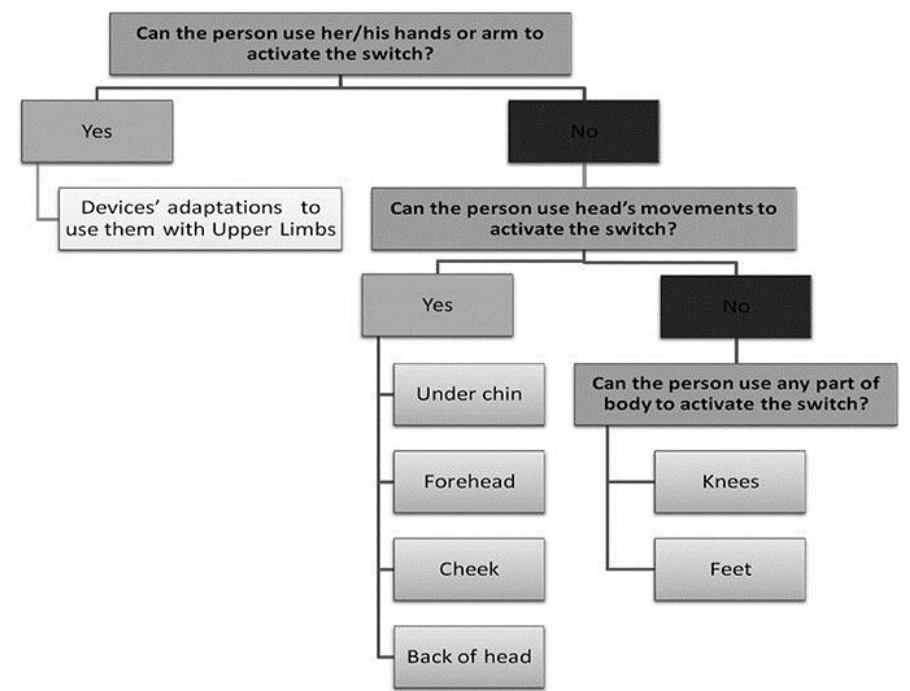


Assessing Mouse Alternatives to Access to Computer: A Case

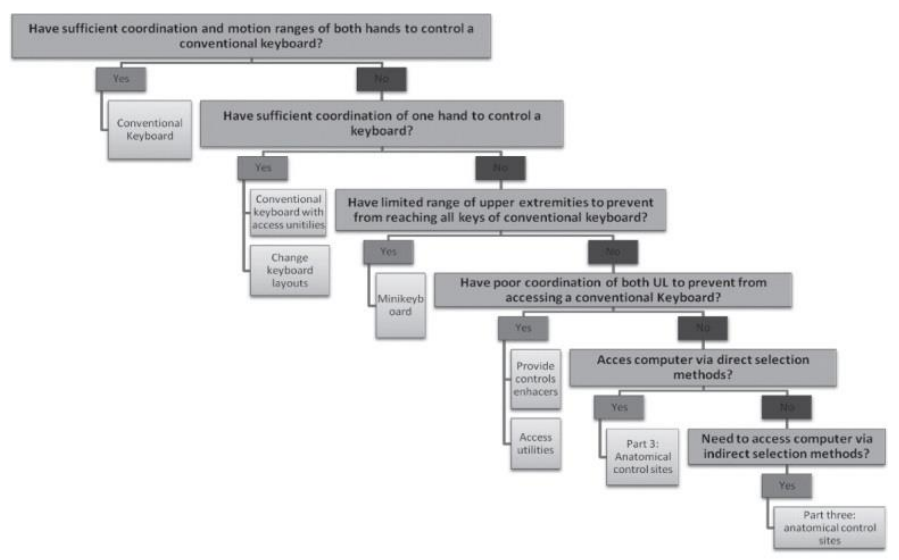

Operate mouse by the right hand?

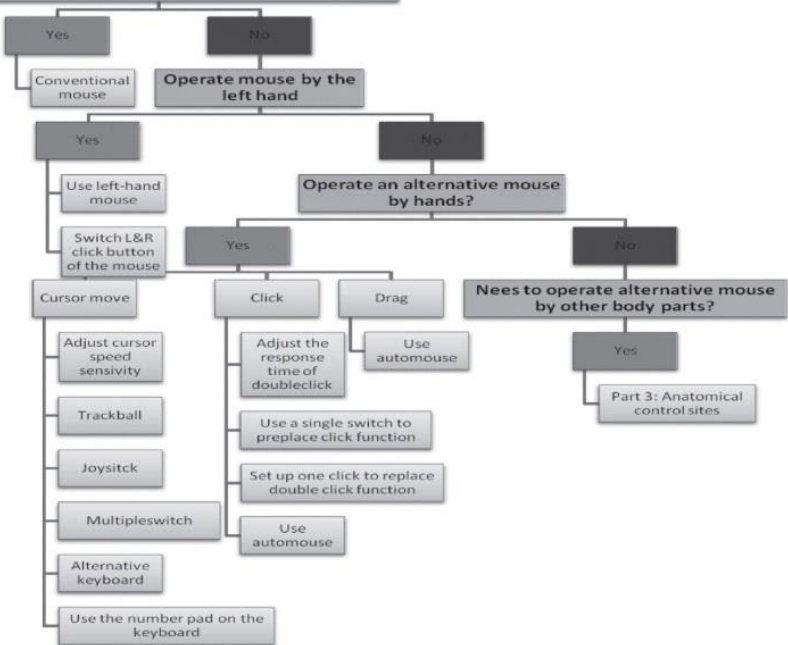

